\title{
An Avian-Inspired Passive Mechanism for Quadrotor Perching
}

\author{
Courtney E. Doyle, Student Member, IEEE, Justin J. Bird, Taylor A. Isom, Jason C. Kallman, Daman F. Bareiss, \\ David J. Dunlop, Raymond J. King, Jake J. Abbott, Member, IEEE, and Mark A. Minor, Member, IEEE
}

\begin{abstract}
Flying robots capable of perch-and-stare are desirable for reconnaissance missions. Inspired by an adaptation that enables songbirds to sleep in trees without active muscle control, the research presented herein details the design for a passive mechanism that enables a rotorcraft to perch reminiscent of a bird perching on a tree branch. Perching is accomplished through the integration of a compliant, underactuated gripping foot and a collapsing leg mechanism that converts rotorcraft weight into tendon tension in order to passively actuate the foot. Analysis of mechanism behavior is presented, and stability tests were performed to characterize the ability of the system to reject disturbances. The results indicate that it is possible to passively perch a rotorcraft on multiple surfaces and support reasonable environmental disturbances. The analysis in this paper can enable passive perching design optimization in vertical take-off and landing systems.
\end{abstract}

Index Terms-Bioinspired, grasp, passive actuation, perch-andstare, tendon, underactuation.

\section{INTRODUCTION}

$\mathbf{P}$ ERCH-AND-STARE describes a maneuver in which a robot flies to a vantage point and lands to collect intelligence. A robot capable of perch-and-stare, equipped with cameras and other sensors, can provide an inconspicuous reconnaissance platform. Additionally, robot perching provides a potential means for recharging the robot's power source (e.g., through the use of photovoltaics).

Our work aims to provide a landing mechanism that expands the number of surfaces upon which a rotorcraft can land. Inspired by the perching capability of songbirds (order Passeriformes), we present a design that can perch on a variety of surfaces [see Fig. 1(a)]. Songbirds exhibit a particulary interesting adaptation that equips them for life in the trees [1], [2]. As depicted in

Manuscript received November 1, 2011; revised April 15, 2012; accepted July 8, 2012. Date of publication August 31, 2012; date of current version January 10, 2013. Recommended by Guest Editor M. Sitti. This work was supported in part by the University of Utah and in part by the National Science Foundation under Grant DGE-0654414. This paper was presented in part at the 2011 IEEE/RSJ International Conference on Intelligent Robots and Systems, San Francisco, CA, September 25-30.

The authors are with the Department of Mechanical Engineering, University of Utah, Salt Lake City, UT 84112, USA. (e-mail: courtney.doyle@ utah.edu; jake.abbott@utah.edu; mark.minor@utah.edu).

This paper has supplementary downloadable material available at http://ieeexplore.ieee.org, provided by the author. The material consists of a video, viewable with Windows Media Player, demonstrating the ability of the avian-inspired passive mechanism to: 1) support a quadrotor under external disturbances and 2) perch from flight using a mock-flight/control set-up. The size of the video is 33.7 MB. Contact courtney.doyle @ utah.edu for further questions about this work.

Color versions of one or more of the figures in this paper are available online at http://ieeexplore.ieee.org.

Digital Object Identifier 10.1109/TMECH.2012.2211081
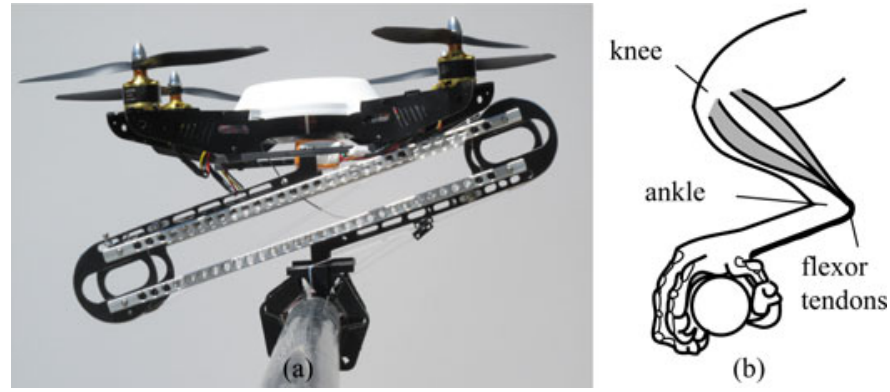

(b)

Fig. 1. Passive perching concept. (a) Quadrotor perching on a cylindrical railing. The grip is actuated by the weight of the quadrotor. (b) Songbirds have evolved a useful adaptation that allows for sleeping while perching. As the bird folds its legs, the tendons on the rear side of the ankle automatically grip the toes around the branch. This enables the relaxed leg of a sleeping bird to tightly grip the perch without any voluntary muscle effort. Figure recreated based on an image in [3].

Fig. 1(b), songbirds have a tendon on the rear side of the ankle that automatically causes the toes to grip around a branch when the leg bends. This tendon enables songbirds to sleep while perching without active control of gripping. As a bird relaxes, the weight of its body causes the legs to bend and the toes to grip; deeper relaxation leads to a tighter grip. When the bird is ready to take off, it must actively stand up to let go of the perch. This anatomy is key to the development of our passively actuated system; the weight of the rotorcraft is used to actuate the gripping mechanism. When the rotorcraft lifts off, the foot will naturally release.

In addition, birds' feet are underactuated, meaning they have more degrees of freedom (dof) than muscles to control them, which enables the feet to passively conform to the structure on which they are attempting to perch. Underactuated grippers are a focus of study for many research groups, and designs have been developed that utilize passive mechanical components such as compliant mechanisms [4]-[9] and mechanical limits [4], [6], [7], as well as clever differential transmissions [4], [6]-[11]. A number of groups have developed designs that utilize four bar mechanisms or gears to transmit force and move the fingers [4], [5], [10], [11]; however, more related to our study are underactuated hands driven by tendons. One of the earliest systems was developed by Hirose and Umetani consisting of a multilink, two-fingered mechanism driven by a pair of wires (flexor and extensor) wrapped around successively smaller pulleys [12]. A similar design was used at Northeastern University to develop Graspar, a three-fingered hand capable of grasping a variety of objects [13]. Other groups have employed a single tendon for flexing while utilizing springs for a restoring force [6]-[8]; 
the group at Université Laval is capable of controlling a 15dof hand with a single motor [6]. Of particular interest to us is an underactuated design of Dollar and Howe in which an 8dof four-fingered hand is controlled using a single actuator [9]. Their design uses compliant joints between stiff link segments with a cable secured at the distal links to transmit actuation force, and is shown to be capable of grasping objects of varying size and shape. To date, a single study has investigated robot gripping inspired by the foot of a bird. Researchers at Clemson University analyzed the feet of raptors, motivated by their excellent grasping ability and simple design relative to anthropomorphic hands [14]. They went on to design a "thumbless" grasping hand reminiscent of a raptor foot [15], but most of the joints have independent actuation.

A number of research groups have pursued solutions to robot perching. Researchers at the University of Florida and colleagues [16] developed a fixed-wing robot with crawling legs, designed to land on a relatively large target zone, such as the top of a building, and then use its legs to crawl to the edge of the building. Researchers at Drexel University [17] equipped a miniature rotorcraft with optic-flow and ultrasonic sensors, enabling the robot to find and land on the edge of a building. Researchers at Cornell University [18] are incorporating a morphing structure inspired by the wings and tail of a bird during landing. Researchers at MIT [19] have developed a method to perform a high-speed maneuver with a fixed-wing robot, such that the robot pulls up into a high angle-of-attack to perch on a string via a latching hook. Researchers at the US Air Force Academy [20] have developed two concepts, one where the aircraft rests over an open space, suspended by four anchors, and the second where a plane uses a sticky pad to adhere to a vertical surface. Researchers at Ecole Polytechnique Fédérale de Lausanne [21] use impact-driven microspines to adhere to inclined and vertical surfaces. Researchers at Stanford University [22] have used a high angle-of-attack approach to attach a fixed-wing aircraft to a vertical surface. They use a compliant, bio-inspired leg-type mechanism as a suspension system between the aircraft and foot that uses microspines for adhesion. Other study from this lab [23] utilizes compliant, underactuated structures for supporting a wall-climbing robot. Researchers at the University of Pennsylvania [24] have used actuated, compliant microspines on a quadrotor for landing on vertical and horizontal surfaces. Each of these methods shares the need for a specific landing surface; the ultimate goal of our avian-inspired approach is to offer perching on the same variety of surfaces available to birds. Currently, our design performs best on horizontal objects with a cross section smaller than the foot, enabling an encircling grasp.

Recently, a group at Yale University attached an underactuated hand to the underside of a helicopter [25]. Their study focused on picking up objects and the effect on flight dynamics, but landing is still accomplished using traditional landing skids.

There has not been any investigation of bird-feet-inspired graspers for perching, other than our preliminary study [26]. In that work, we presented an initial prototype of a compliant leg mechanism, cut from a single piece of material, that actuated an underactuated compliant gripper. The initial prototype was experimentally shown to be capable of perching on cylindrical surfaces. However, the design suffered from limited cyclic life, and its behavior deviated from the idealized model. In this paper, we present a new design with increased stability and cyclic life, more predictable behavior, and capability of perching on a variety of surfaces. It is believed that the original design is better suited for small-scale applications, as forces will be smaller, reducing the negative aspects, and the design presented herein is difficult to produce at small scales. Beyond the extension of our previous analysis to the new design, this paper provides an analysis of foot motion and its mapping to tendon displacement and tension, as well as a stability evaluation of the system. The current design is a proof of concept, and the dimensions are not optimized for any particular rotorcraft. In fact, the rotorcraft used herein was added after the design was complete only to illustrate the functionality of the mechanism.

\section{PASsive LeG MECHANISM}

This section presents a design iteration for the leg mechanism that improves upon our previous work by increasing stability and cyclic life. The leg consists of a knee and ankle joint and three rigid segments: the thigh, shin, and heel. The key difference is that the single-material compliant joints have been replaced with pin joints; this reduces fatigue, reduces out-of-plane motion, generates more idealized behavior, and enables a more exact system analysis. A parallelogram linkage for the shin, with pin joints vertically aligned, enforces that the two joints deflect at the same rate and that the aircraft body keeps a constant orientation. An inelastic cable is used to actuate the attached foot (see Section III). The term tendon is used throughout this paper in keeping with the bio-inspired design, but should not be confused for an elastic material.

The design must satisfy two requirements: 1) rotorcraft center of mass (COM) must stay relatively centered over the foot; and 2) tendon path length must increase as the mechanism collapses. The first idea accounts for system stability during the landing maneuver and the second idea is imperative for passive actuation. Design for the first requirement is presented in Section II-A and design for the second requirement is presented in Section II-B. Design for mechanical advantage is presented in Section II-C.

\section{A. Centering Rotorcraft Mass During Vertical Motion}

As the rotorcraft lowers onto the perch, its COM must stay centered over the foot and the leg should not exert a moment at the foot that causes it to move out from under the body. Studying the free-body diagram presented in Fig. 2 provides design rules that must be followed to enforce proper motion. First, summing forces in the $y$-direction, and assuming the vertically aligned pin joints support equal forces, $F_{w}=\frac{1}{2} F_{y 1 a}=$ $\frac{1}{2} F_{y 1 b}=\frac{1}{2} F_{y 2 a}=\frac{1}{2} F_{y 2 b}=F_{p}$. Second, summing forces in the $x$-direction, $F_{x 1 a}=F_{x 1 b}=F_{x 2 a}=F_{x 1 b}$. Finally, performing a moment balance on links 1 and 3 and solving for $L_{1}$ and $L_{3}: L_{1}=\left(F_{x 1 a} p\right) /\left(F_{w} \cos \phi\right)$ and $L_{3}=\left(F_{x 2 b} p\right) /\left(F_{p} \cos \phi\right)$. Substituting the force balances results in the design requirement, $L_{1}=L_{3}=L$. The force/moment balance does not place requirements on $L_{2}$, but in order to keep the rotorcraft COM 


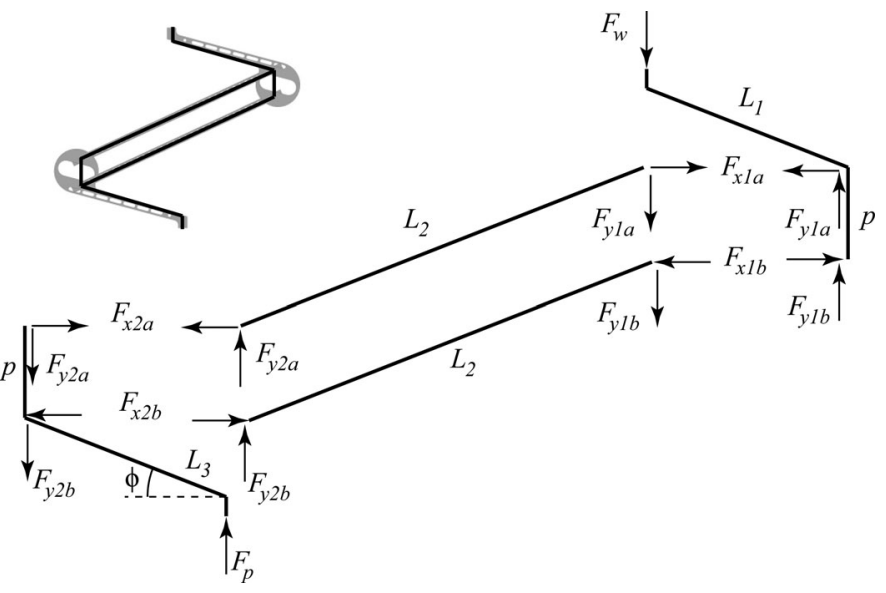

Fig. 2. Free-body diagram of the leg. Inset depicts the full leg with link segments indicated.

over the foot $L_{2}=2 L$. A different value for $L_{2}$ could be used if the leg is not aligned below the rotorcraft's COM; however, this would impose a moment at the top of the leg, which would alter the moment balance of the system and change the requirements on $L_{1}$ and $L_{3}$.

The position of the rotorcraft mounting point, relative to the foot, is dependent on the angular deflection of each joint [see Fig. 3(a)]. Placing the origin at the foot, the location of each joint and the aircraft body are determined as follows:

$$
\begin{aligned}
& A=\left(A_{x}, A_{y}\right)=\left(-L \cos \phi, L \sin \phi+\frac{p}{2}\right) \\
& B=\left(B_{x}, B_{y}\right)=\left(A_{x}+2 L \cos \alpha, A_{y}+2 L \sin \alpha\right) \\
& C=\left(C_{x}, C_{y}\right)=\left(B_{x}-L \cos \phi, B_{y}+L \sin \phi+\frac{p}{2}\right)
\end{aligned}
$$

where $\alpha=\theta-\phi$.

In order to minimize the horizontal motion of the aircraft during collapse, a min-max optimization on $\left|C_{x}\right|$ of (1) is run with respect to $\phi$ over a desirable range of motion for each joint $\theta$. For the range $0^{\circ} \leq \theta \leq 40^{\circ}$, the optimal value of $\phi$ is 16.5 . Fig. 4(a) shows the horizontal deviation for $\phi=16.5^{\circ}$, $L=152.4 \mathrm{~mm}$ and $p=38.1 \mathrm{~mm}$; the values of $L$ and $p$ are the dimensions used in our prototype, which were arbitrarily chosen to create a mechanism roughly scaled for a quadrotor that was able to actuate our foot. The vertical motion of the rotorcraft is shown in Fig. 4(b).

\section{B. Vertical Motion to Tendon Pull}

The tendon routing through the leg can be visualized in Fig. 3(b). The change in tendon path length is dependent on two factors: a wrapping of the tendon around each cam, and a change in length of the tangent between the two cams. By designing the two cams to be symmetric, the model reduces to two sets of identical triangles that intersect at a common point, as seen in Fig. 5(a). Studying a single set of triangles [see Fig. 5(b)] the tendon path length $t$ is determined by summing the arc length of the tendon in contact with the cam, $t_{\mathrm{arc}}=2 r(3 \pi / 2-\gamma)$, and

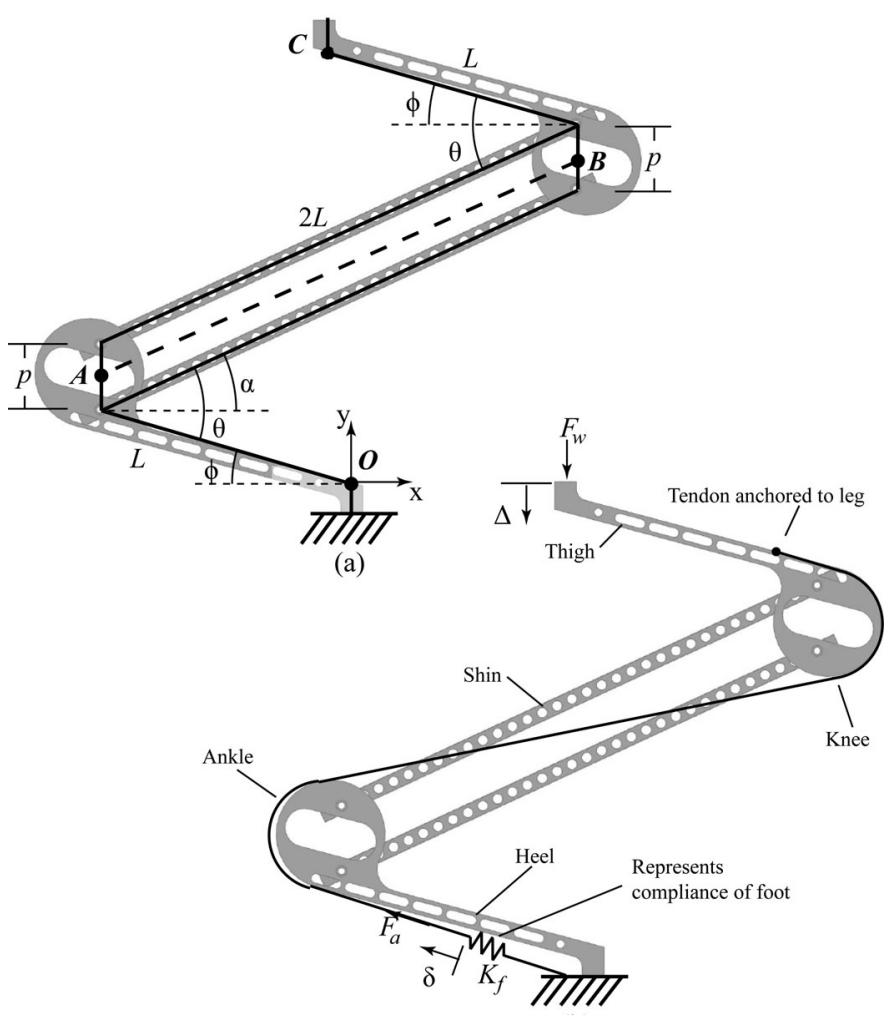

(b)

Fig. 3. Leg models superimposed on a SolidWorks rendering. (a) Geometric model. $L$ is the length of the heel/thigh links, $p$ is the distance between the joints of the parallel shin links, $\theta$ is the angle between links, $\alpha$ is the angle between the shin and horizontal, and $\phi$ is the fixed angle between the heel/thigh and horizontal. $\boldsymbol{A}$ and $\boldsymbol{B}$ are the pivot points located half way between the physical pin joints of the shin links, $\boldsymbol{O}$ is the origin where the foot attaches to the leg, and $\boldsymbol{C}$ is the point at which the aircraft attaches to the leg. The exact locations of $\boldsymbol{O}$ and $\boldsymbol{C}$ are arbitrary, but once selected all other parameters are referenced from their location. (b) Energy balance model. $F_{w}$ is the weight of the rotorcraft, $F_{a}$ is the tension applied to the foot, $\Delta$ is the change in height of the rotorcraft measured from the point when the foot begins to close, $\delta$ is the change in tendon path length just above the foot, and $K_{f}$ is the effective spring constant of the foot (there is no physical spring in series with the cable, other than the compliant foot).

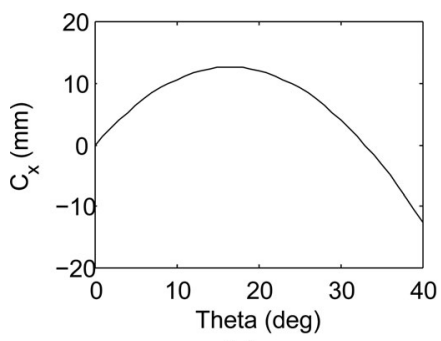

(a)

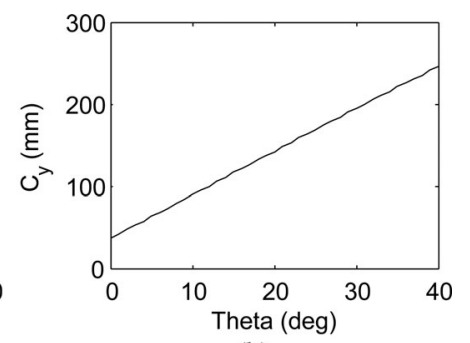

(b)
Fig. 4. Rotorcraft motion during collapse. $\phi=16.5^{\circ}, L=152.4 \mathrm{~mm}$, and $p=38.1 \mathrm{~mm}$. (a) Horizontal shift from center. (b) Height above the foot.

the length of the tangent line between the two cams $T$, as

$$
t=2 r\left(\frac{3 \pi}{2}-\gamma\right)+T
$$

$t_{\mathrm{arc}}$ is defined assuming that the tendon wraps $3 / 4$ of the cam when $\gamma=0^{\circ}$; although the tendon will actually contact slightly less than $3 / 4$ of the circumference, we are concerned with the 


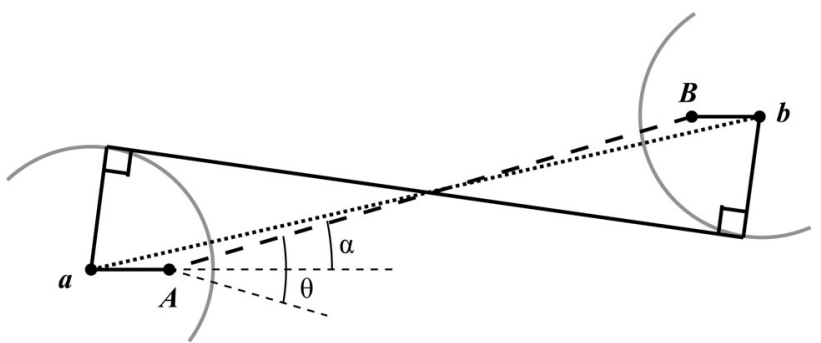

(a)

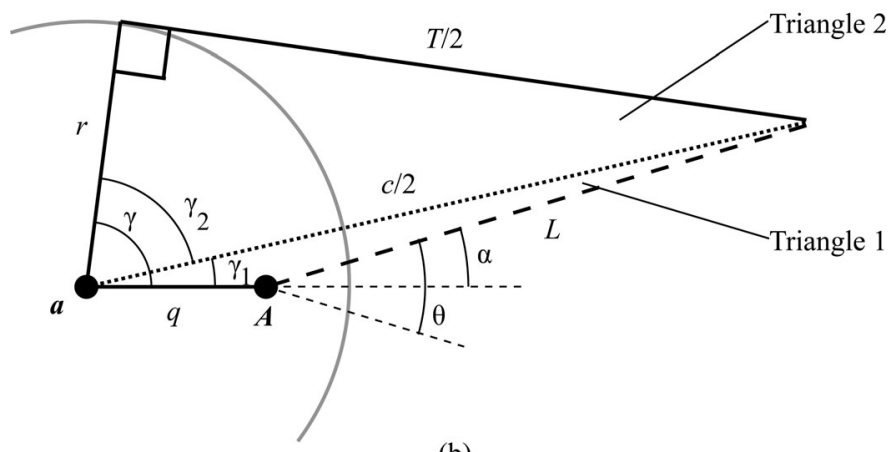

(b)

Fig. 5. Geometry of tendon path length. (a) Total length from joint to joint. (b) Detail view of half the system. $\theta, \alpha, L, \boldsymbol{A}$, and $\boldsymbol{B}$ are as defined previously. $\boldsymbol{a}$ and $\boldsymbol{b}$ are the centers of the circular cams, $c$ is the distance between the center of the cams, $T$ is the distance between the tangent points at which the tendon leaves each cam, $r$ is the radius of each cam, $q$ is the distance between the rotation point and the center of each cam (assumed to be horizontal), and $\gamma, \gamma_{1}$, and $\gamma_{2}$ are defined as shown.

change in path length $\delta$, not the actual length $t$, so this approximation does not affect the final result.

The task now lies in determining values for $\gamma$ and $T$. Examining Triangle 1 , trigonometric identities can be used to first determine $c$ and then $\gamma_{1}$

$$
\begin{aligned}
\left(\frac{c}{2}\right)^{2} & =q^{2}+L^{2}-2 q L \cos (\pi-\alpha) \\
\frac{\sin \gamma_{1}}{L} & =\frac{\sin (\pi-\alpha)}{c / 2} .
\end{aligned}
$$

Next, examining Triangle 2, geometric and trigonometric identities can be used to determine $T$ and $\gamma_{2}$

$$
\begin{aligned}
r^{2}+\left(\frac{T}{2}\right)^{2} & =\left(\frac{c}{2}\right)^{2} \\
\tan \gamma_{2} & =\frac{T / 2}{r} .
\end{aligned}
$$

Finally, the angle $\gamma$ is known, as the sum of $\gamma_{1}$ and $\gamma_{2}$. The two parameters of interest are thus:

$$
\begin{aligned}
T= & 2 \sqrt{q^{2}+L^{2}-r^{2}+2 q L \cos (\theta-\phi)} \\
\gamma= & \sin ^{-1}\left(\frac{L \sin (\theta-\phi)}{\sqrt{q^{2}+L^{2}+2 q L \cos (\theta-\phi)}}\right) \\
& +\tan ^{-1}\left(\frac{T}{2 r}\right) .
\end{aligned}
$$

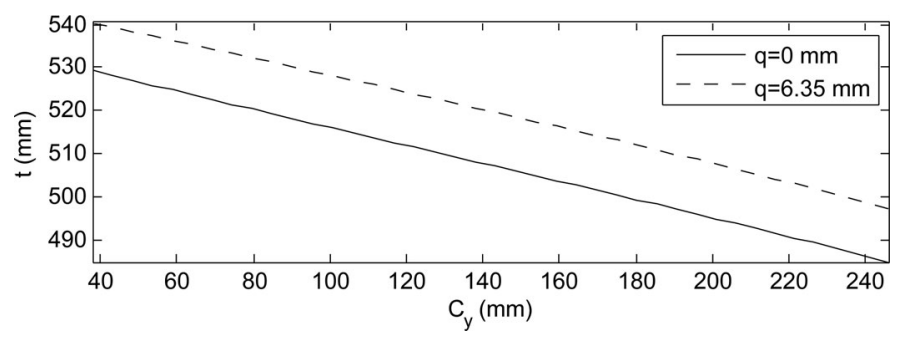

Fig. 6. Tendon path length versus vertical displacement. As the leg collapses, tendon path length increases. The total effect on tendon travel $\delta$ due to $q$ is insignificant $(<3 \%)$ for $\phi=16.5^{\circ}, L=152.4 \mathrm{~mm}, p=38.1 \mathrm{~mm}$, and $r=31.7 \mathrm{~mm}$

A plot showing how the tendon path length changes with vertical motion is presented in Fig. 6, where the values of $\phi, L$, and $p$ are chosen as before and $r=31.7 \mathrm{~mm}$ is the value used in our prototype, which was chosen to provide an appropriate amount of tendon travel based on our prototype foot. Plots for both $q=6.35 \mathrm{~mm}$, which is the value on our prototype, and $q=0 \mathrm{~mm}$ are shown. Subsequent analysis becomes difficult with $q \neq 0$ due to noninvertible functions, so $q=0$ is used to further analyze the system; the error introduced in $\delta$ is minimal $(<3 \%)$, as demonstrated by Fig. $6 . T$ and $\gamma$ are simplified with $q=0$, and the tendon path length $t$ is described as follows:

$$
t=2 r\left(\frac{3 \pi}{2}-\tan ^{-1}\left(\frac{\sqrt{L^{2}-r^{2}}}{r}\right)+\theta-\phi\right)+2 \sqrt{L^{2}-r^{2}}
$$

\section{Rotorcraft Weight to Tendon Tension}

The amount of tension available to close the gripper is related to the weight of the rotorcraft. Performing an energy balance on the system shown in Fig. 3(b)

$$
F_{w} \Delta=\frac{1}{2} K_{f} \delta^{2}
$$

Considering the leg collapsed by some nominal value $\Delta$, collapsing the system by a differential change $d \Delta$ produces

$$
F_{w}(\Delta+d \Delta)=\frac{1}{2} K_{f}\left(\delta^{2}+2 \delta d \delta+d \delta^{2}\right) .
$$

Substituting in (10), recognizing that $d \delta^{2}$ can be neglected, and substituting in the locally linear relationship $F_{a}=K_{f} \delta$, (11) can be solved for the mechanical advantage of the system $F_{a} / F_{w}$, which relates the weight of the rotorcraft to tendon tension at the foot. Note that $d C_{y}=-d \Delta$ and $d t=d \delta$, so

$$
\frac{F_{a}}{F_{w}}=\frac{d \Delta}{d \delta}=-\frac{d C_{y}}{d t} .
$$

Thus, the mechanical advantage of the system is found by differentiating $C_{y}$ with respect to $t$.

$C_{y}$ of (1) can be expanded as

$$
C_{y}(\theta)=2 L \sin (\theta-\phi)+2 L \sin \phi+p .
$$




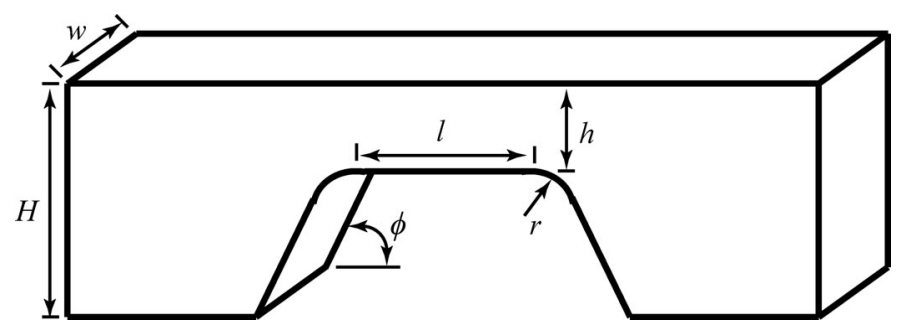

Fig. 7. Parameters characterizing joint geometry. $w$ is the width of the toe, $H$ is the height of the toe, $h$ is the height of the joint, $l$ is the length of the joint, $r$ is the radius of the joint fillet, and $\phi$ is the notch angle.

But we need $C_{y}(t)$, not $C_{y}(\theta)$, so solving (9) for $\theta$ gives the following:

$$
\theta=\frac{3 \pi}{2}+Q-\tan ^{-1} Q+\phi-\frac{t}{2 r}
$$

where $Q=\sqrt{L^{2}-r^{2}} / r$. Thus, substituting (14) into (13), we obtain

$$
C_{y}(t)=2 L \sin \left(\frac{3 \pi}{2}+Q-\tan ^{-1} Q-\frac{t}{2 r}\right)+2 L \sin \phi+p .
$$

Differentiating (15) with respect to $t$ produces the following:

$$
\frac{d C_{y}}{d t}=-\frac{L}{r} \cos \left(\frac{3 \pi}{2}+Q-\tan ^{-1} Q-\frac{t}{2 r}\right)
$$

which can be written with respect to $\theta$ as

$$
\frac{d C_{y}}{d t}=-\frac{L}{r} \cos (\theta-\phi) \text {. }
$$

The mechanical advantage has a minimum value of 4.4 when the leg is fully open and a maximum value of 4.8 approximately half way through collapse.

\section{UNDERACTUATED GRIPPING FOOT}

A desirable characteristic of the bird foot is the underactuated structure. This allows the toes to passively conform to unknown surface shapes. Our foot is a simplified variation on [9]. Using a waterjet, individual toes were cut from a sheet of polyurethane (McMaster-Carr Item 8716K736), using notches to create flexible joints. Hollow tubing was attached to the underside of each toe segment for tendon routing. This simple design, using a single material, was chosen for ease of manufacturing and scalability. Design of the foot is presented first by characterizing the joint in Section III-A, then analyzing motion in Section III-B.

\section{A. Joint Characterization}

Each toe joint (see Fig. 7) can be classified as a small-length flexural pivot and is represented by its pseudo-rigid-body model as a pin joint with a torsion spring of stiffness $K=E I / l_{0}$, where $E$ is the Young's modulus, $I$ is the cross-sectional moment of inertia, and $l_{0}$ is the flexible segment length [27]. Because our flexible segment does not have a uniform cross section, due to the notch taper, validation of this model was necessary. By hanging known weights from samples with varying parameters, we were able to investigate the instantaneous center of rotation (ICR) and

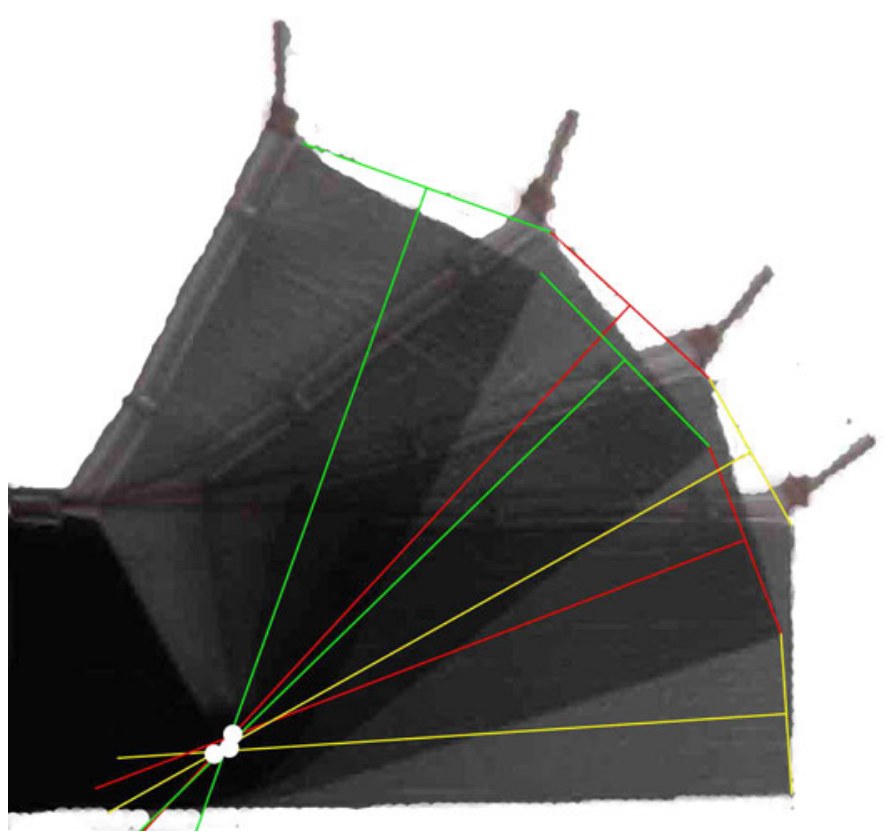

Fig. 8. Instantaneous center of rotation (ICR) does not vary significantly as a joint deflects. Image overlays joint at four deflection positions. The ICR, designated by the white dots, is determined by drawing the perpendicular bisectors of the line segments connecting similar points between deflections.

stiffness of each joint. It was found that the ICR of individual joints do not vary significantly (see Fig. 8) and that stiffness is linearly related to the geometric term $w h^{3} / l$. Therefore, the pseudo-rigid-body model is valid for representing joint motion.

Combining joints in series produces a toe structure that can passively comply to a surface. The stiffness of each joint was determined by two central requirements: 1) for the largest workspace, and thus largest range of gripping capability, it is desirable for the joints to deflect in order from proximal to distal; and 2) each joint should be able to return to some nominal deflection with no applied tendon tension.

The first requirement can be satisfied by enforcing that $K_{1}<$ $K_{2}<\ldots<K_{n}$, as demonstrated in Fig. 9, where joint 1 is the most proximal. Notice how the gripping ability changes for each joint pattern. The top row shows a toe with the proposed pattern, $K_{1}<K_{2}<K_{3}$. The toe makes contact with the object starting with the first segment, followed by the second and then third, making a successful closure. The middle row shows a toe with the pattern $K_{1}=K_{2}=K_{3}$. For the perch shown, the toe is able to make a closure, but each segment makes contact at roughly the same time. For an unsymmetrical perch this toe will not be able to grip reliably. The bottom row shows a toe with the pattern $K_{1}>K_{2}>K_{3}$. This toe experiences significant deflection at the third joint first, which causes the toe to miss closure around the perch.

To satisfy the second requirement, we enforce $K_{1}>K_{1 \mathrm{~min}}$, where $K_{1 \mathrm{~min}}$ is determined by the weight of the distal portion of the toe supported by the first joint and a desired nominal deflection. Because the first joint will experience the most weight, and each successive joint is stiffer than the previous, it follows that each joint satisfies the second requirement so long as the 


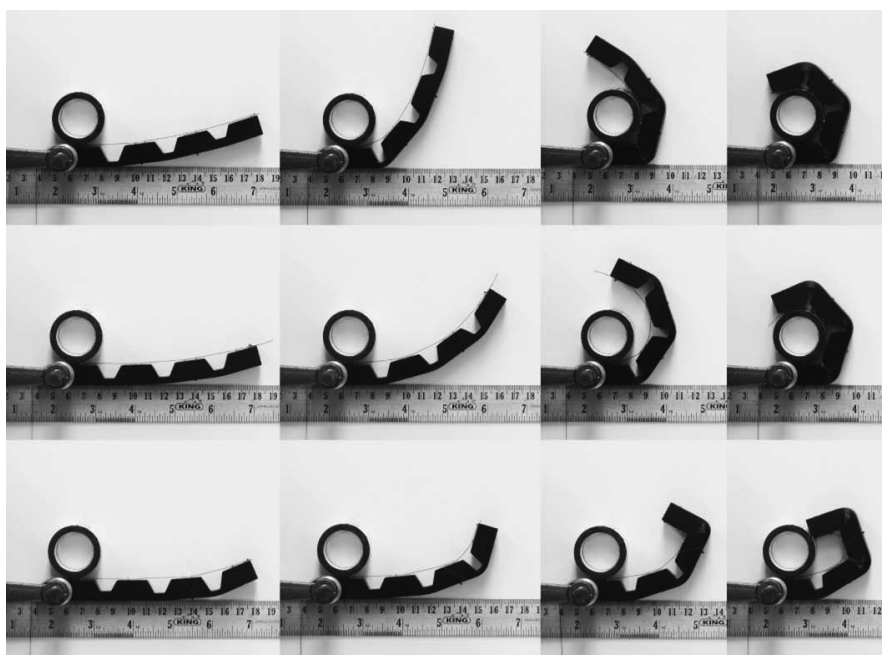

Fig. 9. Depiction of how joint stiffness patterns affect form closure. (Top row) $K_{1}<K_{2}<K_{3}$; (Middle row) $K_{1}=K_{2}=K_{3}$; (Bottom Row) $K_{1}>$ $K_{2}>K_{3}$.

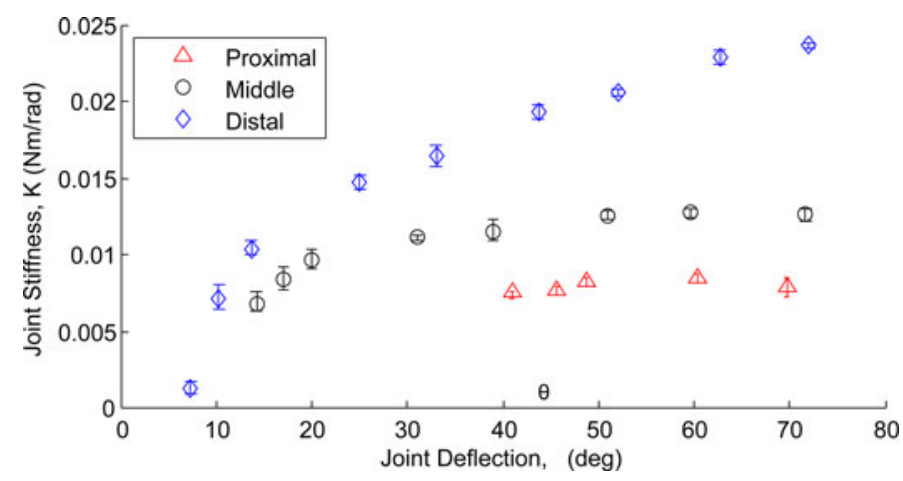

Fig. 10. Joint stiffness as a function of deflection. Values are calculated using the mean joint deflections. Error bars show the range of $K$ values possible with all permutations of $\pm \sigma$ in joint deflection, where $\sigma$ is the standard deviations of recorded joint deflections. Stiffness is nonlinearly related to deflection and increases as the toe deflects further.

first joint does. The above constraints were used to create three identical toes, which are attached to the leg using an adaptation of the anisodactyl arrangement.

The stiffness component of the pseudo-rigid-body model was initially assumed constant, following the work of [27]. The model, however, was derived under the assumption that the flexible member experienced pure bending; this is not a valid assumption in our toes; thus, the stiffness must be derived. A single toe was mounted such that the first link was horizontal and the distal links sagged under their own weight. Calibrated weights were hung from the tendon, using a pulley to convert vertical motion of the weight into horizontal tendon pull, and the deflection of each joint was recorded. The process was repeated three times to analyze repeatability. The subsequent joint stiffness is calculated using the recursive formula presented next, and can be visualized in Fig. 10. Notice that the stiffness increases nonlinearly as the joints deflect through larger angles.

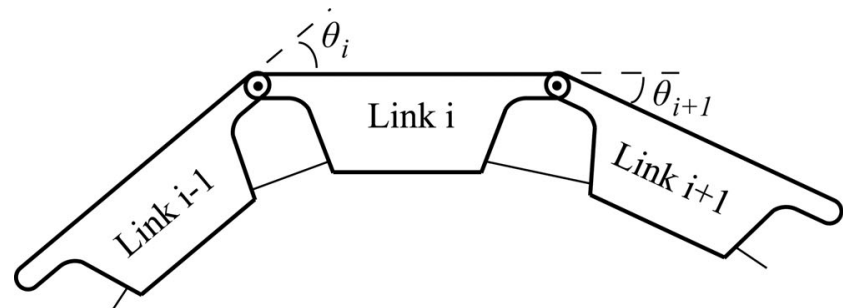

(a)

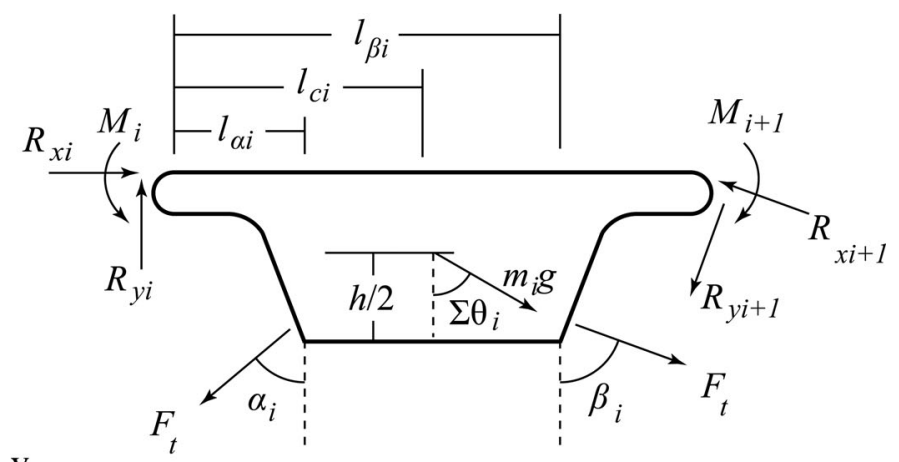

$\mathrm{y}_{\uparrow}$

(b)

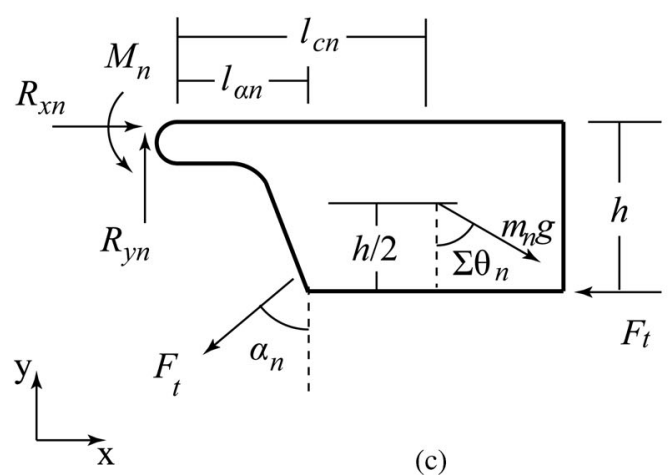

Fig. 11. Toe model for deflection through free space. (a) Parameter and geometric labeling of toe. $\theta_{i}$ is the angular displacement of link $i$ with respect to link $i-1$. (b) Free body diagram of link $i . M_{i}$ is the moment of joint $i, m_{i}$ is the mass of link $i, g$ is the gravitational constant, $l_{c i}$ is half the link length, where the mass is applied, $\Sigma \theta_{i}$ is the sum of all joint angles from $\theta_{1}$ to $\theta_{i}, F_{t}$ is the tendon tension, $\alpha_{i}$ and $\beta_{i}$ are the tendon departure angles from link $i$, and $l_{\alpha}$ and $l_{\beta}$ are the moment arms through which the tendon forces are applied. (c) Free body diagram of link $n$, which is the most distal. $h$ is the moment arm through which the tendon is attached and the remaining parameters are as defined before.

\section{B. Joint Motion}

Utilizing the joint model, we can study a free-body diagram of the toe to predict motion and relate it to tendon displacement as well as tendon tension (see Fig. 11). With this analysis, we are neglecting the friction due to tendon movement and assuming only tension components in the $y$-direction act on the intermediate links. Furthermore, it can be shown that $\alpha_{i}=\pi / 2-\theta_{i} / 2$ and $\beta_{i}=\pi / 2-\theta_{i+1} / 2$. Finally, for each joint, $M_{i}=K_{i} \theta_{i}$. Performing moment and force balances, if joint deflection and tendon tension are known, joint stiffness can be determined, as depicted in Fig. 10. The recursive formula for joint stiffness is 


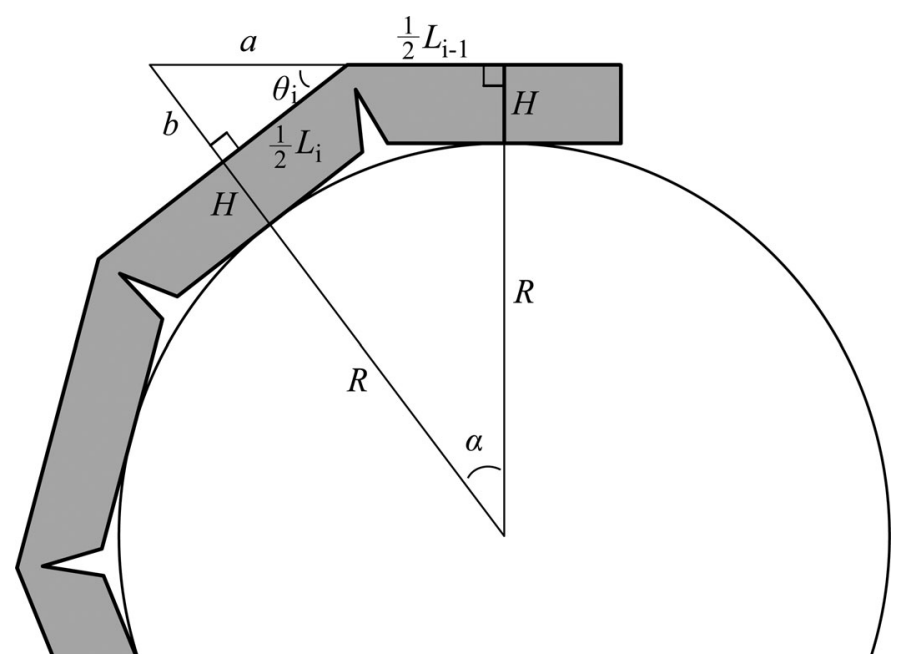

Fig. 12. Model used to determine toe deflection to grip a cylindrical perch. $H$ is the height of the toe, $L_{i}$ is the length of link $i, R$ is the radius of the perch, $\theta_{i}$ is the deflection of joint $i$, and $\alpha$ is the angle between the contact points of link $i$ and $i-1$.

presented in (18)-(23). Alternatively, if joint stiffness and tendon tension are known, joint deflection can be determined by rearranging the equations and iteratively stepping through the recursion until an equilibrium deflection is reached.

$$
\begin{aligned}
K_{n}= & \frac{1}{\theta_{n}}\left(F_{t}\left(l_{\alpha n} \sin \left(\frac{\theta_{n}}{2}\right)+h\right)\right. \\
& \left.+m_{n} g\left(l_{c n} \cos \Sigma \theta_{n}-\frac{h}{2} \sin \Sigma \theta_{n}\right)\right) \\
R_{x n}= & F_{t}-m_{n} g \sin \Sigma \theta_{n} \\
R_{y n}= & F_{t} \sin \left(\frac{\theta_{n}}{2}\right)+m_{n} g \cos \Sigma \theta_{n} \\
K_{i}= & \frac{1}{\theta_{i}}\left(F_{t}\left(l_{\alpha i} \sin \left(\frac{\theta_{i}}{2}\right)+l_{\beta i} \sin \left(\frac{\theta_{i+1}}{2}\right)\right)\right. \\
& +2 l_{c i}\left(R_{y i+1} \cos \theta_{i+1}-R_{x i+1} \sin \theta_{i+1}\right) \\
& +m_{i} g\left(l_{c i} \cos \Sigma \theta_{i}-\frac{h}{2} \sin \Sigma \theta_{i}\right) \\
& \left.+K_{i+1} \theta_{i+1}\right) \\
R_{x i}= & R_{x i+1} \cos \theta_{i+1}+R_{y i+1} \sin \theta_{i+1} \\
& -m_{i} g \sin \Sigma \theta_{i} \\
R_{y i}= & F_{t}\left(\sin \left(\frac{\theta_{i}}{2}\right)+\sin \left(\frac{\theta_{i+1}}{2}\right)\right)+m_{i} g \cos \Sigma \theta_{i} \\
& +R_{y i+1} \cos \theta_{i+1}-R_{x i+1} \sin \theta_{i+1}
\end{aligned}
$$

We now present an analysis to determine the joint deflections required to grip a circular cross section of a particular radius $R$, which can be used to determine the necessary displacement of the actuating tendon. Studying Fig. 12, two similar triangles are

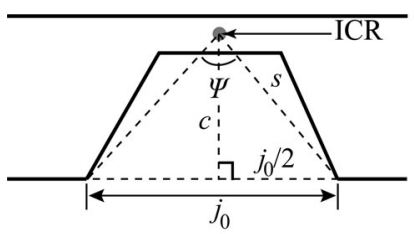

(a)

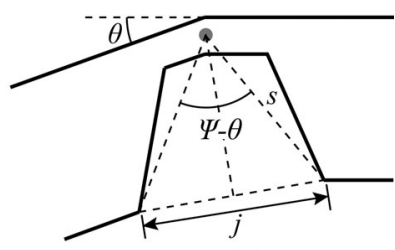

(b)
Fig. 13. Model used to determine tendon pull required to deflect a joint. (a) Toe in undeflected shape. (b) Toe in deflected shape. $j_{0}$ is the size of the undeflected joint notch where the tendon is routed, $j$ is the size of the deflected joint notch, $c$ is the distance from the tendon to the ICR, and $\theta$ is the joint deflection.

formed with equal angles $\theta_{i}=\alpha$. From the larger triangle,

$$
\begin{aligned}
\cos \alpha & =\frac{R+H}{R+H+b} \\
\tan \alpha & =\frac{a+L_{i-1} / 2}{R+H} .
\end{aligned}
$$

From the smaller triangle,

$$
\begin{aligned}
\cos \theta_{i} & =\frac{L_{i}}{2 a} \\
\tan \theta_{i} & =\frac{2 b}{L_{i}} .
\end{aligned}
$$

Equating (24) with (26) and (25) with (27) produces the system of equations that can be solved for $b$, which is substituted into (27) and solved for $\theta_{i}$

$$
\theta_{i}=\tan ^{-1}\left(\frac{2}{L_{i}} \frac{\left(L_{i}^{2}+L_{i-1} L_{i}\right)(R+H)}{4(R+H)^{2}-L_{i}^{2}}\right) .
$$

The amount that the tendon must be pulled to create these deflections is now calculated by studying the joint geometry shown in Fig. 13. First, the initial values $\psi$ and $s$ must be determined through geometry from Fig. 13(a)

$$
\begin{gathered}
\psi=2 \tan ^{-1}\left(\frac{j_{0}}{2 c}\right) \\
s=\sqrt{\frac{j_{0}^{2}}{4}+c^{2}} .
\end{gathered}
$$

Next, the new gap length is calculated through geometry from Fig. 13(b)

$$
j=2 s \sin \left(\frac{\psi-\theta}{2}\right) .
$$

Finally, the required tendon pull $\delta$ can be calculated as the difference between the two notch lengths, and summed over each joint

$$
\delta=\sum_{i=1}^{n}\left(j_{0 i}-j_{i}\right)
$$

where $j_{0 i}$ is the original notch length and $j_{i}$ is the deflected notch length of joint $i$. 


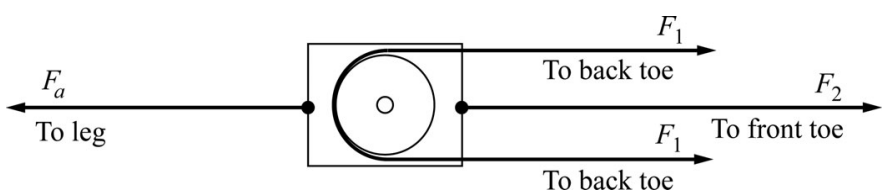

Fig. 14. Differential system applied to tendons, where $F_{1}$ and $F_{2}$ are the tendon tensions in individual toes and $F_{a}$ is the tendon tension applied by the leg.

\section{COMPONENT INTEGRATION}

For maximum grasping adaptability, it is desirable that each toe be able to move semi-independently. A pulley block is utilized to create differential actuation of each toe (see Fig. 14). The tendon routed from the leg is tied to one side of the pulley mount and the front toe is tied to the opposite side of the mount. The two back toes are connected by a single tendon, routed around the pulley. The tendon in each toe will displace at equivalent rates through open space, providing symmetric closure. If motion in one of the back toes is constrained, the pulley system will allow the remaining toes to move $\delta$ and $2 \delta$ for the front and back toe, respectively. Furthermore, through open space, this setup creates an equal force distribution among each of the toes, such that $F_{1} \approx F_{2} \approx F_{a} / 3$. This is an approximate relationship because the tendon does not necessarily depart the pulley in a direction parallel to $F_{a}$; deviation will cause slight variations in tension. Once motion in one back toe is constrained, the two back toes will still experience equivalent tension, but the tension in the tendon of the back toes will increase at twice the rate of the front toe due to the change in displacement rates.

The tendon material is a fused superline $(10 \mathrm{lb}$ Berkley FireLine Fused Crystal), which has high strength with minimal stretch. A soft Buna-N foam (McMaster-Carr, Item \# 93745K21) and coarse sandpaper (60 grit emery cloth) was applied to the surface of each toe segment to provide greater friction at the grip. Additionally, two mechanical pin-type stops were added to each cam to restrain the motion of the leg when freely dangling beneath the rotorcraft.

Two leg mechanisms were attached to a Gaui 330X Quad Flyer. The quadrotor weighs $388 \mathrm{~g}$ and has a payload of $700 \mathrm{~g}$, which must include a battery. After attaching our mechanisms, we witnessed an undesirable amount of independent actuation between the two legs, which caused instability in perching. To mitigate this, we added two stabilizers connecting the legs at the heel and thigh, which caused the legs to move more dependently. Because of compliance in the joints, the legs can still collapse to slightly different heights, providing the ability to perch on surfaces of different cross sections. The completed two-leg mechanism weighs $478 \mathrm{~g}$, which leaves $222 \mathrm{~g}$ for batteries and future additions such as cameras or GPS systems. For reference, the $2200 \mathrm{mAh}$ lithium polymer battery we are using weighs $145 \mathrm{~g}$, which means there is $77 \mathrm{~g}$ remaining in the payload; this value will likely need to be increased, which would require reducing the weight of the leg and foot. The complete system can be visualized in Fig. 15.

\section{EXPERIMENTAL Stability EVAluation}

The functional quality of the landing gear was studied through four experiments. First, we studied the ability of the landing gear

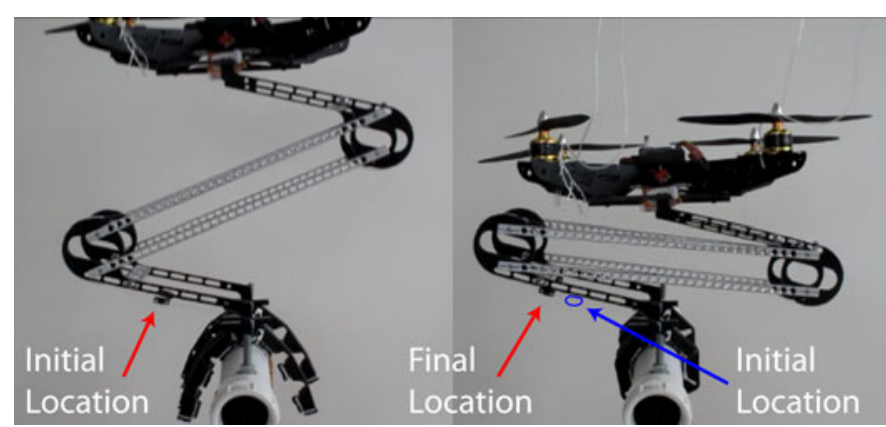

Fig. 15. Mechanism in initial (Left) and final (Right) perching states. The pulley is used to indicate the tendon motion.

to support a quadrotor while perching on a variety of surfaces. Second, we studied the ability of the landing gear to support a quadrotor under angular perturbations from vertical. Third, we studied the ability of the landing gear to support the quadrotor under lateral disturbances. For the first three experiments, the system was manually placed on the perches. A fourth test explored perching using a mock flight setup.

\section{A. Versatile Perching Capability}

To understand the versatility of the landing gear, the system was perched on a variety of commonly found surfaces. The system is able to perch on many types of surfaces, but performs best on surfaces that allow the toes to wrap around the perch. The cylindrical railing in Fig. 1 is similar to the PVC tubing used to test the mechanism during development. Similarly, the system is able to perch on a tree branch, Fig. 16(a), which is roughly cylindrical but not homogeneous in geometry and texture. Fig. 16(b) shows the system perching on a rectangular railing, where the foot is just large enough to grip around the front of the surface; qualitatively, this perch is remarkably stable, and the sharp corners seem to act to lock the toes in place. On more oddly shaped surfaces, the system can succeed in perching; however, its stability is less predictable. For example, Fig. 16(c) and (d) shows the system perching on exposed plumbing; the perch shown is at equilibrium, but slight angular deviations of the quadrotor resulted in unstable perches. Notice that the system is not only able to stabilize on perches with uniform foot stance [see Fig. 16(c)], but also on perches with nonuniform foot stance [see Fig. 16(d)]. Fig. 16(e) shows the system on a rock with nonuniform topography.

Additionally, we are interested in how the system lands on flat surfaces. As shown in Fig. 16(f), the legs tilt forward and the system comes to a resting position on the feet and the knee joints. Although this orientation may not be ideal, it is repeatable and stable. The ability of a rotorcraft to take off from this position will need to be investigated before determining if this is an acceptable landing position.

\section{B. Stability Under Angular Perturbations}

It is unreasonable to expect a robotic rotorcraft to be able to land on the perch perfectly vertically. When perched at an off-center angle, the full rotorcraft weight is not available to actuate the gripper, and a portion acts to pull the system out of 


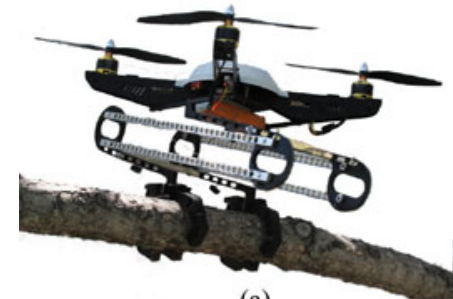

(a)

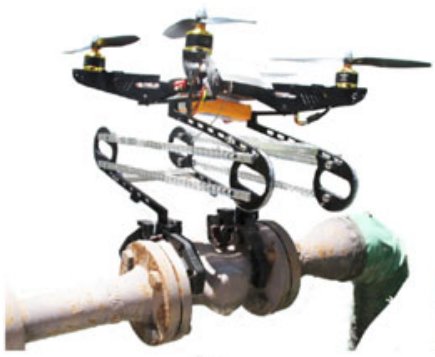

(c)

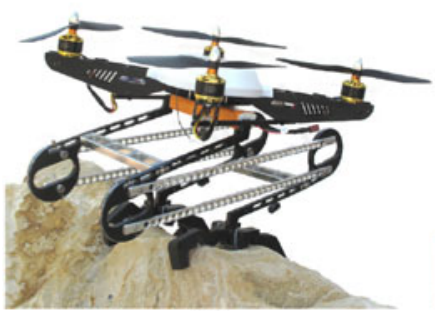

(e)

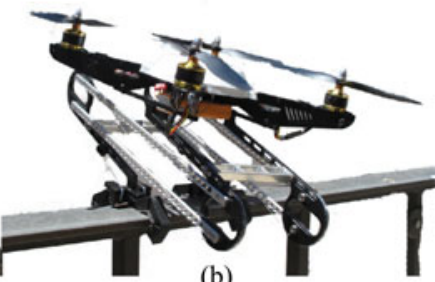

(b)

(d)

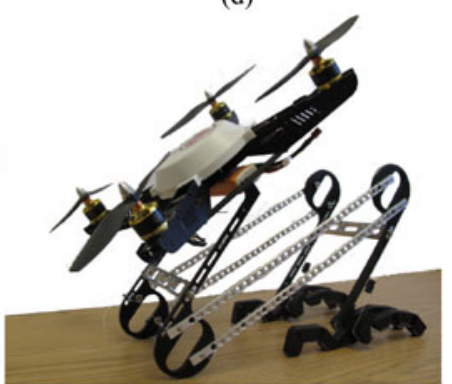

(f)

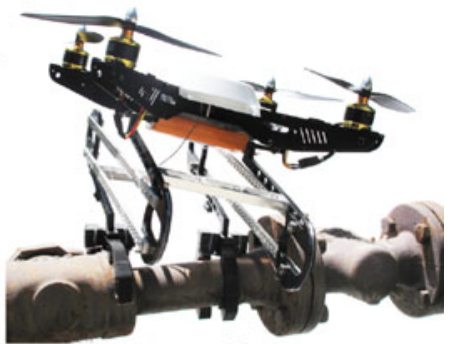

Fig. 16. Successful perches on common objects. (a) Tree branch. (b) Rectangular railing. (c) Plumbing, with similar foot stances. (d) Plumbing, with asymmetrical foot stances. (e) Ridge of rock. (f) Flat ground.

equilibrium. As such, we explored the range of angles at which the system is stable. The experimental setup consisted of a perch that could freely rotate, with an Arduino Mega microcontroller and a GMS high-torque servo motor to control rotation of the perch (see Fig. 17). With the servo initialized at $0^{\circ}$, the landing mechanism and rotorcraft were centered on the perch; the servo then stepped through rotation in increments of $1^{\circ}$ and held for $30 \mathrm{~s}$. The point at which the landing mechanism released was recorded. Twenty readings were averaged to quantify variance. This method was performed for both positive and negative angles on painted PVC perches of 33- and 49-mm diameters, where a positive angle is defined as one that tilts the front of the rotorcraft toward the ground. The results are summarized in Table I. At large angles $\left(\gtrsim 10^{\circ}\right)$, the servo motor was not able to completely overcome the moment imposed on the perch and often sat at a slightly larger angle than that being commanded; thus, the data reported here are a conservative estimate. It is also important to note that the values reported here are sensitive to the tendon tensioning.

There are two important trends to notice from the data. First, the system has a higher tolerance to angular perturbations on the smaller perch; this is because the quadrotor sits closer to the perch, so the COM acts at a smaller moment arm than on the larger perch. As such, it may be beneficial to determine characteristic minimal perch size for a given application, then design

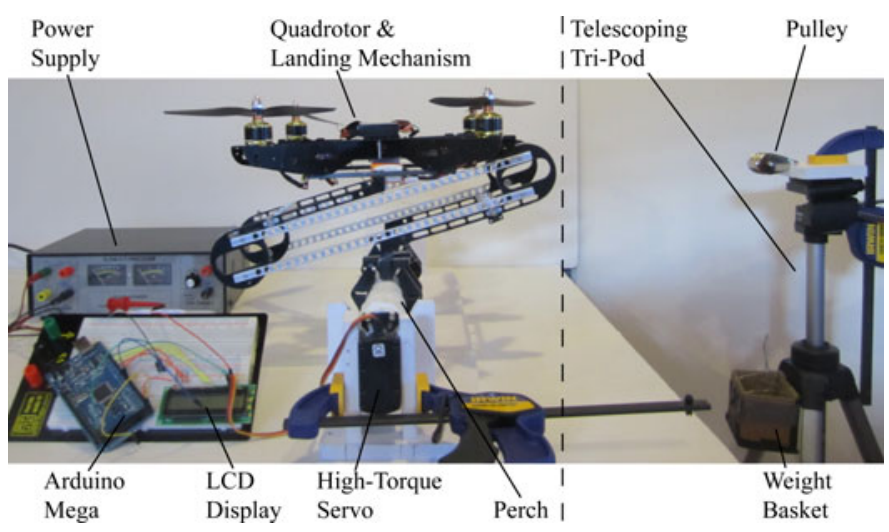

Fig. 17. Perturbation test setup. (Left) Angular perturbation test setup. (Right) Additional components for lateral perturbation test.

TABLE I

ANGULAR STABILITY LIMITS

\begin{tabular}{|c|c|c|c|}
\hline & \multicolumn{3}{|c|}{$\begin{array}{c}33 \text { mm Painted PVC } \\
\text { Mean } \pm \mathrm{StDev}^{\circ}\end{array}$} \\
\hline Material & Sandpaper & Silicone Rubber & PolyVinyl Mesh \\
\hline Neg. Limit & $-13.5 \pm 1.2$ & - & - \\
\hline Pos. Limit & $11.9 \pm 1.3$ & - & - \\
\hline & \multicolumn{3}{|c|}{$\begin{array}{c}49 \text { mm Painted PVC } \\
\text { Mean } \pm \text { StDev }^{\circ}\end{array}$} \\
\hline Material & Sandpaper & Silicone Rubber & PolyVinyl Mesh \\
\hline Neg. Limit & $-11.6 \pm 1.4$ & $-6.9 \pm 1.0$ & $-10.4 \pm 1.7$ \\
\hline Pos. Limit & $9.3 \pm 1.3$ & $4.7 \pm 1.8$ & $11.7 \pm 2.0$ \\
\hline & \multicolumn{3}{|c|}{$\begin{array}{c}42 \text { mm Fence Rail } \\
\text { Mean } \pm \text { StDev }^{\circ}\end{array}$} \\
\hline Material & Sandpaper & Silicone Rubber & PolyVinyl Mesh \\
\hline Neg. Limit & $-7.3 \pm 1.2$ & $-6.8 \pm 1.2$ & $14.25 \pm 1.9$ \\
\hline Pos. Limit & $4.7 \pm 1.6$ & $5.7 \pm 1.4$ & $14.05 \pm 1.9$ \\
\hline
\end{tabular}

the leg so that the aircraft sits low for that size perch. Second, the system has a higher tolerance to angular perturbations in the negative direction; this may be due to the structure of the leg. For instance, when the leg collapses, the shin links are at an angle such that the knee is lower than the ankle; this means that as the aircraft tilts forward (positive angles) the pitch of the shin is exaggerated and the COM of the leg produces a higher moment. However, when the aircraft tilts backward (negative angles), the shin links approach horizontal and the COM of the legs produces a lower moment.

As an initial investigation of grip materials, we repeated the test on a 42- mm diameter commercial fence toprail and included three additional grip materials reported to have good surface friction characteristics: a natural rubber (McMaster-Carr Item 93625K248), a silicone rubber (McMaster-Carr Item 5781T48), and a polyvinyl foam mesh (McMaster-Carr Item 85695K1). The new materials were also tested on the 49-mm PVC perch. The results are summarized in Table I. Data for the natural rubber are not included in the table because it performed worse than the original sandpaper material. From the data, it appears that the polyvinyl mesh is a better material for gripping ability. Further tests for durability should be performed before making a final selection. 
TABLE II

LATERAL FORCE LIMITS

\begin{tabular}{|c|c|c|c|}
\hline & \multicolumn{3}{|c|}{$\begin{array}{c}33 \text { mm Painted PVC } \\
\text { Mean } \pm \text { StDev }\end{array}$} \\
\hline Perch Angle & $-7^{\circ}$ & $0^{\circ}$ & $6^{\circ}$ \\
\hline \multirow{5}{*}{$\begin{array}{lc}\text { Neg. Limit } & (\mathrm{N}) \\
& (\mathrm{Nm}) \\
\text { Pos. Limit } & (\mathrm{N}) \\
& (\mathrm{Nm}) \\
\end{array}$} & $-1.7 \pm 0.1$ & $-3.0 \pm 0.1$ & $-3.7 \pm 0.2$ \\
\hline & $-0.23 \pm 0.02$ & $-0.40 \pm 0.02$ & $-0.50 \pm 0.03$ \\
\hline & $3.9 \pm 0.2$ & $2.6 \pm 0.2$ & $1.5 \pm 0.1$ \\
\hline & $0.53 \pm 0.02$ & $0.36 \pm 0.02$ & $0.20 \pm 0.01$ \\
\hline & \multicolumn{3}{|c|}{$\begin{array}{c}49 \text { mm Painted PVC } \\
\text { Mean } \pm \text { StDev }\end{array}$} \\
\hline Perch Angle & $-6^{\circ}$ & $0^{\circ}$ & $5^{\circ}$ \\
\hline \multirow{4}{*}{$\begin{array}{l}\text { Neg. Limit } \\
\text { Pos. Limit }\end{array}$} & $-2.1 \pm 0.2$ & $-3.0 \pm 0.2$ & $-3.8 \pm 0.3$ \\
\hline & $-0.37 \pm 0.04$ & $-0.54 \pm 0.04$ & $-0.69 \pm 0.05$ \\
\hline & $3.5 \pm 0.2$ & $2.9 \pm 0.2$ & $1.5 \pm 0.2$ \\
\hline & $0.62 \pm 0.04$ & $0.52 \pm 0.03$ & $0.27 \pm 0.04$ \\
\hline
\end{tabular}

\section{Stability Under Lateral Disturbances}

Once the system has made a stable perch, it will likely experience environmental disturbances such as wind. In this scenario, the full rotorcraft weight is actuating the gripper, but the lateral force acts to pull the system out of equilibrium. Thus, we designed an experiment to test the amount of horizontal force the system can withstand. The setup from the angular perturbation test was reused, and a pulley was added so that a horizontal force can be applied by hanging weights on a cable (see Fig. 17). The quadrotor was set at angles of $0^{\circ}$ and $1 / 2$ the magnitude of the maximum angular perturbation in the positive and negative directions from Table I for sandpaper on the painted PVC perches. Weight was applied to a cable that was secured to the bottom plate of the quadrotor and held for $30 \mathrm{~s}$. The point at which the feet slipped off the perch was recorded. Ten readings were averaged to quantify variance. This method was performed on perches of 33- and 49-mm diameters for positive and negative forces, where a positive force is one that acts to move the rotorcraft forward. The results are summarized in Table II, with equivalent values for the acting moment disturbance at the foot, calculated as the product of the force and the distance from the center of the perch at which the force is applied (137 and $180 \mathrm{~mm}$ for the 33- and 49-mm perches, respectively).

When perched away from vertical, the most obvious thing to notice is that there is an asymmetry between the disturbances that can be supported in the negative and positive directions. This is as expected. Take, for example, the $33-\mathrm{mm}$ perch at $-7^{\circ}$ : here, a negative force pulls the system in the same direction as gravity and into a larger angle, but a positive force pulls the system against gravity and into a smaller angle.

Although a point force is not the same as a wind gust, these data give an idea of the amount of disturbance that can be supported as it is seen as a moment at the foot. An initial attempt was made to correlate lateral force to an equivalent wind speed. We used the Treadport Adaptive Wind Tunnel (TPAWT) developed at the University of Utah [28]. The TPAWT directs wind flow to augment the experience of a virtual reality participant. With the TPAWT, we were not able to consistently achieve wind speeds that caused our perching mechanism to fail, so a rigorous analysis of the capabilities cannot currently be provided. Reliably, an environment with average wind speeds of the order of $5 \mathrm{~m} / \mathrm{s}$ and gusts up to $6.5 \mathrm{~m} / \mathrm{s}$ could be created; in this
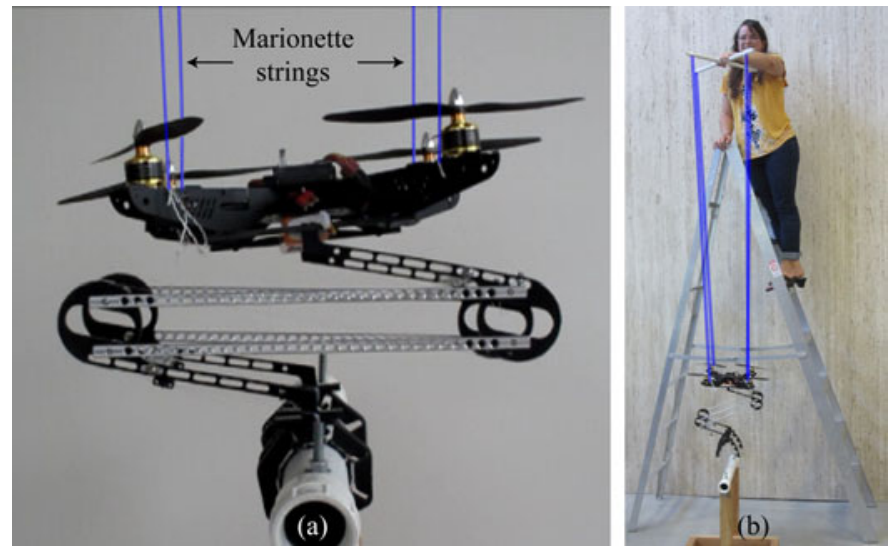

Fig. 18. Mock flight test setup. (a) Marionette strings attached at each motor, maintained parallel to the motor axes. (b) Experimenter guided the quadrotor using a marionette with $1.7 \mathrm{~m}$ strings. Strings are highlighted in blue for clarity.

setting, the quadrotor on the 33-mm painted PVC perch was agitated by the disturbance, but did not release the grip. When failure was induced, gust values varying from 8 to $12 \mathrm{~m} / \mathrm{s}$ were recorded, where the wind vector was clearly a significant factor for stability.

\section{Mock Perching From Flight}

At this time, we do not have autonomous flight control algorithms, so it is not possible to present perching reliability from flight. However, in order to approximate system performance from flight, we set up a rig similar to a marionette. Four $1.7 \mathrm{~m}$ strings, one per motor, were attached to a cross such that, when hanging, the strings pulled in a direction parallel to the propeller axis of rotation. As long as the string stays closely aligned with this axis, any applied force represents an equivalent thrust from the propeller.

Two scenarios were run, each with ten attempts, and the success rate was recorded. A successful perch was defined as one where the quadrotor remained stable on the perch after the guide strings were slack. A total of six trials were run, alternating which scenario was performed first, to determine an average and standard deviation; a 15-min break was taken between each trial in order to reduce human learning and fatigue. Scenario A represents a flight control capable of accurately centering the rotorcraft over the perch, but has no control of the final descent. We lifted the quadrotor off the perch and rested in a hover-like position, while the toes were allowed to bump the perch and stabilize the COM over the perch. The quadrotor was then quickly dropped, meant to represent a shutoff of the motors. Scenario B represents a flight control capable of both centering over the perch and controlling the decent. For the setup, we slowly lowered the rotorcraft from above, allowing adjustments provided the string remained parallel with the rotor rotation axes. The test setup can be visualized in Fig. 18.

Scenario A had a success rate of $73 \pm 9 \%$ and Scenario B had a success rate of $98 \pm 4 \%$. These numbers tell us that if a control algorithm is developed that can closely center the rotorcraft over the perch, the rotorcraft should find a stable perch most of the 
time. If the algorithm is also capable of controlling the decent, the success rate should increase significantly. These results are promising for future development of autonomous flight.

\section{DISCUSSION AND FUTURE WORK}

Results from the stability experiments show that the current, unoptimized design is capable of passively supporting moderate external disturbances. The stability range can be improved by increasing the friction at the grip. Friction can be increased with a better selection of contact material on each toe; an initial study was presented in Section V-B, but a more rigorous study of materials may provide even better results.

Additionally, friction can be increased by increasing the normal force at each contact point, which directly correlates to the mechanical advantage of the system. Future iterations will use an optimized leg design, to provide the best mechanical advantage for our quadrotor. The equations presented in Section II can be used to optimize the leg dimensions for a given foot, balancing required tendon travel with mechanical advantage. The equations in Section III can be used to optimize foot dimensions for a desired application, balancing required toe deflection with tendon pull and joint stiffness.

A concern for grip strength is line-stretch over time. Initially, we used a monofilament fishing line for our tendon material, which experienced significant stretch within the first cycle. We then upgraded to the fused superline, which has minimal stretch. More significant than tendon stretch is slippage at the tensioning screws; improving this connection is a goal for the next prototype. Fortunately, moderate slack in the tendon does not render the mechanism useless. The slack is analogous to backlash in geared systems, requiring the quadrotor to lower a small amount before engaging the gripper, shifting the system on the mechanical advantage curve. As long as the slack is not excessive, the system can still maintain sufficient grasp.

Other topics for future consideration are material selection, altering toe design, studying flight dynamics, and integrating a flight controller. The current leg and foot materials were chosen out of convenience; the current mechanism is within the payload of the rotorcraft, however lighter materials will allow for additional items such as surveying cameras and sensors to be added to the quadrotor. By altering the toe design, both length and number of segments, it is possible that better grips can be achieved. Now that the concept has been shown feasible, the ability to fly with the mechanism attached must be studied. This ultimately will result in the development of a controller for autonomous flight and perching. Current work in autonomous quadrotor flight suggests that a controller can be developed for stable flight. The mock flight conditions presented in Section VD lead us to believe that a controller can be developed to control the landing maneuver.

\section{CONCLUSION}

The prototype presented in this paper demonstrates that robot perching on a variety of surfaces can be achieved through the integration of a passive actuating mechanism with an underactuated gripper. Stability tests demonstrated the range of distur- bances that can be supported. Although this current prototype performs relatively well, improvements can be made in future design iterations. One topic of interest is studying the effects of altering toe length and the number of segments; it is possible that better grips on a wider variety of surfaces can be achieved by altering these characteristics. Also, the current prototype dimensions were chosen somewhat arbitrarily so that the mechanism was scaled approximately to the size of a quadrotor and achieved at least the minimum tendon pull and tension necessary to close the foot; using the governing equations presented herein, more optimal dimensions can be selected to reduce weight and improve performance. Finally, the effect of the landing gear on flight dynamics, and methods to mitigate this effect, remains the most significant open question.

\section{ACKNOWLEDGMENT}

The authors would like to thank C. Johnson and J. Simpson for their contributions to the design of the leg and acknowledge the support of T. Slowik in the Department of Mechanical Engineering. Additionally, they would also like to thank Dr. R. Meyers at Weber State University for fruitful discussions about bird anatomy and function.

\section{REFERENCES}

[1] F. B. Gill, Ornithology, 3rd ed. New York: Freeman, 2007.

[2] N. S. Proctor and P. J. Lynch, Manual of Ornithology: Avian Structure and Function. Ann Arbor, MI: Edwards Brothers, 1993.

[3] E. Britannica. (2011). Bird. [Online]. Available: http://www.britannica. com/EBchecked/topic/66391/bird

[4] T. Laliberté, L. Birglen, and C. M. Gosselin, "Underactuation in robotic grasping hands," Mach. Intell. Robot. Control, vol. 4, no. 3, pp. 1-11, 2002.

[5] M. Doria and L. Birglen, "Design of an underactuated compliant gripper for surgery using nitinol," J. Med. Devices, vol. 3, no. 011007, 2009.

[6] C. Gosselin, F. Pelletier, and T. Laliberté, "An anthropomorphic underactuated robotic hand with 15 dofs and a single actuator," in Proc. IEEE Int. Conf. Robot. Autom., 2008, pp. 749-754.

[7] B. Massa, S. Roccella, M. C. Carrozza, and P. Dario, "Design and development of an underactuated prosthetic hand," in Proc. IEEE Int. Conf. Robot. Autom., 2002, pp. 3374-3379.

[8] C. Cho, Y. Lee, and M. Kim, "Underactuated hand with passive adaptation," in Proc. IEEE Int. Symp. Ind. Electron., 2009, pp. 995-1000.

[9] A. M. Dollar and R. D. Howe, "The highly adaptive SDM hand: Design and performance evaluation," Int. J. Robot. Res., vol. 29, pp. 585-597, 2010.

[10] M. Luo, T. Mei, X. Wang, and Y. Yu, "Grasp characteristics of an underactuated robot hand," in Proc. IEEE Int. Conf. Robot. Autom., vol. 3, 2004, pp. 2236-2241

[11] B. Almasri and F. B. Ouezdou, "New design of one motor driven under actuated humanoid hand," in Proc. IEEE/RSJ Int. Conf. Intell. Robots Syst., 2007, pp. 1491-1496.

[12] S. Hirose and Y. Umetani, "The development of soft gripper for the versatile robot hand," Mechanism Mach. Theory, vol. 3, pp. 351-359, 1978.

[13] J. D. Crisman, C. Kanojia, and I. Zeid, "Graspar: A flexible, easily controllable robotic hand," IEEE Robot. Autom. Mag., vol. 3, no. 2, pp. 32-38, Jun. 1996.

[14] A. M. Ramos and I. D. Walker, "Raptors-inroads to multifingered grasping," in Proc. IEEE/RSJ Int. Conf. Intell. Robots Syst., 1998, pp. 467-475.

[15] A. M. Ramos, I. A. Gravagne, and I. D. Walker, "Goldfinger: A nonanthropomorphic, dextrous robot hand," in Proc. IEEE Int. Conf. Robot. Autom., 1999, pp. 913-919.

[16] R. J. Bachmann, F. J. Boria, R. Vaidyanathan, P. G. Ifju, and R. D. Quinn, "A biologically inspired micro-vehicle capable of aerial and terrestrial locomotion," Mechanism Mach. Theory, vol. 44, pp. 513-526, 2009.

[17] T. W. Danko, A. Kellas, and P. Y. Oh, "Robotic rotorcraft and perch-andstare: Sensing landing zones and handling obscurants," in Proc. IEEE Int. Conf. Adv. Robot., 2005, pp. 296-302. 
[18] A. M. Wickenheiser and E. Garcia, "Optimization of perching maneuvers through vehicle morphing," J. Guid., Control, Dyn., vol. 31, no. 4, pp. 815823, 2008.

[19] R. Cory and R. Tedrake, "Experiments in fixed-wing UAV perching," in Proc. AIAA Guid., Navigat., Control Conf., 2008, pp. 1-12.

[20] M. L. Anderson, C. J. Perry, B. M. Hua, D. S. Olsen, J. R. Parcus, K. M. Pederson, and D. D. Jensen, "The sticky-pad plane and other innovative concepts for perching UAVs," in Proc. AIAA Aerospace Sci. Meeting, 2009, pp. 460-470.

[21] M. Kovač, J. Germann, C. Hürzeler, R. Y. Siegwart, and D. Floreano, "A perching mechanism for micro aerial vehicles," J. Micro-Nano Mechatronics, vol. 5, no. 4, pp. 77-91, 2010.

[22] A. L. Desbiens, A. T. Asbeck, and M. R. Cutkosky, "Landing, perching and taking off from vertical surfaces," Int. J. Robot. Res., vol. 30, no. 3, pp. 355-370, 2011.

[23] S. Kim, M. Spenko, S. Trujillo, B. Heyneman, D. Santos, and M. Cutkosky, "Smooth vertical surface climbing with directional adhesion," IEEE Trans. Robot., vol. 24, no. 1, pp. 65-74, Feb. 2008.

[24] D. Mellinger, M. Shomin, and V. Kumar, "Control of quadrotors for robust perching and landing," in Proc. Int. Powered Lift Conf., 2010, pp. 119-126.

[25] P. E. I. Pounds and A. M. Dollar, "Hovering stability of helicopters with elastic constraints," in Proc. ASME Dyn. Syst. Control Conf., 2010, pp. 781-788.

[26] C. E. Doyle, J. J. Bird, T. A. Isom, C. J. Johnson, J. C. Kallman, J. A. Simpson, R. J. King, J. J. Abbott, and M. A. Minor, "Avian-inspired passive perching mechanism for robotic rotorcraft," in Proc. IEEE/RSJ Int. Conf. Intell. Robots Syst., 2011, pp. 4975-4980.

[27] L. L. Howell, Compliant Mechanisms, 1st ed. New York: Wiley, 2001.

[28] S. D. Kulkarni, M. A. Minor, E. R. Pardyjak, and J. M. Hollerbach, "Combined wind speed and angle control in a virtual environment," in Proc. IEEE/RSJ Int. Conf. Intell. Robots Syst., 2008, pp. 1005-1010.

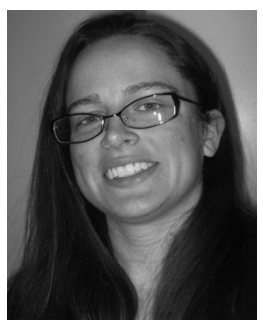

Courtney E. Doyle (S'10) received the B.S. degrees in mechanical and electrical engineering from the University of Michigan, Ann Arbor, in 2007, and the M.S. degree in mechanical engineering from the University of Utah, Salt Lake City, in 2011, with work focusing on the bio-inspired grasping mechanisms. She was awarded an IGERT Traineeship from NSF for Biocentric Robotics at the University of Utah, where she is currently working the Ph.D. degree in mechanical engineering, studying human-robot interaction related to emotions and expression.

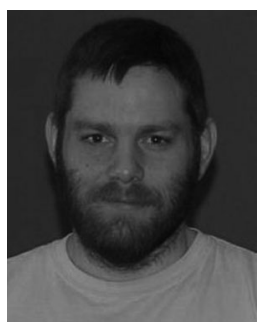

Justin J. Bird received the B.S. degree in mechanical engineering from the University of Utah, Salt Lake City, in 2011.

$\mathrm{He}$ is currently working in the Kennecott Copper Division of Rio Tinto.

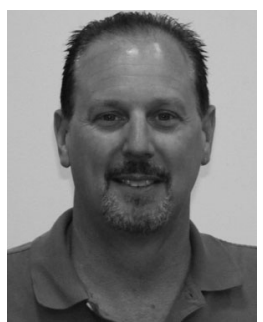

Taylor A. Isom received the B.S. degree in mechanical engineering from the University of Utah, Salt Lake City, in 2011.

$\mathrm{He}$ is currently working as an automation engineer at Bard Access Systems.

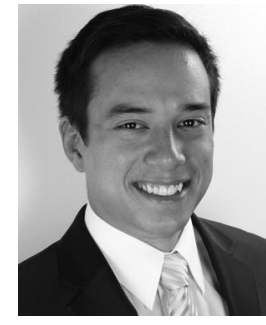

Jason C. Kallman received the B.S. degree in mechanical engineering from the University of Utah, Salt Lake City, in 2011.

$\mathrm{He}$ is currently working as a 3D modeler at K'nowbe Works, LLC.

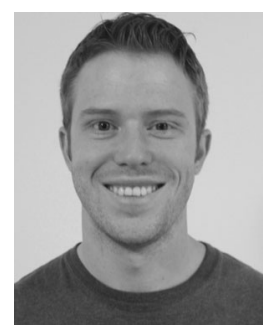

Daman F. Bareiss received the B.S. degree in mechanical engineering in 2011 from Oklahoma State University, Stillwater. He was awarded an ARCS Foundation scholarship and an IGERT Traineeship from the NSF for Biocentric Robotics at the University of Utah, Salt Lake City, UT, where he is currently working toward the Ph.D. degree in mechanical engineering, investigating the interactions between humans and highly maneuverable, human-ridden mobile robots.

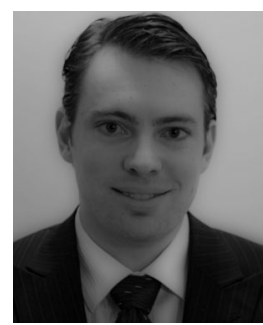

David J. Dunlop received the B.S. and M.S. degrees in mechanical engineering from the University of Utah, Salt Lake City, in 2010. He performed research in the field of precision machine design. He was awarded an IGERT Traineeship from the NSF for Biocentric Robotics at the University of Utah, where he is currently working toward the $\mathrm{Ph} . \mathrm{D}$. degree in mechanical engineering, studying quadrupedal robotic gait.

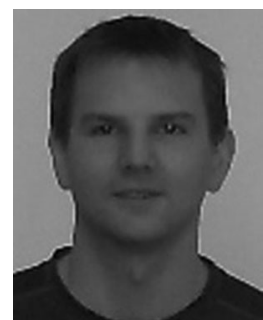

Raymond J. King received the B.S. and M.S. degrees in engineering science and mechanics from Virginia Tech, Blacksburg, in 2009 and 2010, respectively. He is currently working toward the Ph.D. degree in mechanical engineering in the University of Utah, Salt Lake City, where his work consists of modeling the tendon system of the human hand.

He was the recipient of the IGERT Traineeship from NSF for Biocentric Robotics, University of Utah.

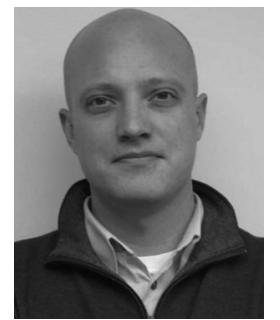

Jake J. Abbott (M'06) received the B.S. degree from Utah State University, Logan, in 1999, the M.S. degree from the University of Utah, Salt Lake City, in 2001, and the Ph.D. degree from The Johns Hopkins University, Baltimore, MD, in 2005, all in mechanical engineering.

In 2005, he became a Postdoctoral Research Associate at ETH Zurich, Switzerland. In 2008, he became an Assistant Professor in the Department of Mechanical Engineering, University of Utah, where he is currently the Head of the Telerobotics Laboratory.

Dr. Abbott is the recipient of the NSF Faculty Early Career Developmen Award, and the 2010 ICRA Best Manipulation Paper Award.

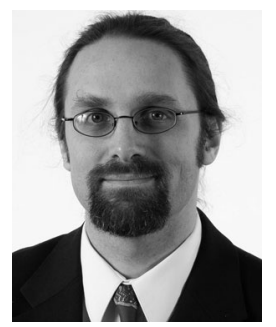

Mark A. Minor (M'00) received the B.S. degree in mechanical engineering from the University of Michigan, Ann Arbor, in 1993, and the M.S. and $\mathrm{Ph} . \mathrm{D}$. degrees in mechanical engineering from Michigan State University, East Lansing, in 1996 and 2000, respectively.

He is currently an Associate Professor in the Department of Mechanical Engineering, University of Utah, Salt Lake City, where he has been a faculty member since 2000. His research interests focus on design and control of robotic systems including mobile robots, rolling robots, climbing robots, flying robots, autonomous ground vehicles, and virtual reality systems. 